\title{
Acute popliteal artery thrombogenesis after total knee arthroplasty: a case report
}

\section{Ri Liu(D582321529@qq.com )}

The second hospital of TangShan https://orcid.org/0000-0002-7626-4622

\section{Lishu Gao}

Tangshan people's hospital

\section{Haiqiang Wei}

Peking University People's Hospital

\section{Kunnan Xie}

The second hospital of TangSahn

\section{Research article}

Keywords: Total knee arthroplasty, Acute popliteal artery thrombogenesis, Post-operative complications

Posted Date: February 27th, 2020

DOI: https://doi.org/10.21203/rs.2.24707/v1

License: (9) This work is licensed under a Creative Commons Attribution 4.0 International License. Read Full License 


\section{Abstract}

Total knee arthroplasty (TKA) is a type of artificial joint replacement surgery. An acute lower limb arterial embolism after TKA is extremely rare. Although the incidence of acute lower extremity popliteal artery thrombogenesis is very low, orthopedists need to be alert to the complication because it may lead to amputation and other serious consequences. We report a 65-year-old man who had 38 years of posttraumatic pain in the left knee joint, which has worsened in the past 3 years, thus limiting mobility. He had an acute popliteal artery thrombogenesis after undergoing a TKA. Although orthopedic and vascular surgeons took active measures in a timely fashion, the patient still had lower extremity dysfunction. After a TKA, patients should be actively instructed to perform rehabilitation exercises to prevent thromboses.

\section{Introduction}

Total knee arthroplasty (TKA) is a type of artificial joint replacement surgery. TKA refers to a treatment method that excises the joint surface that the body cannot self-repair, replaces the damaged joint with artificial joint components, corrects the force line of the limbs, eliminates the pain in the knee joint, maintains the stability of the joint, and recovers the function of the knee joint. The application of TKA is increasing rapidly year-after-year worldwide. Currently, TKA is the most effective method for the treatment of advanced osteoarthritis, rheumatoid arthritis, and other musculoskeletal diseases.

An acute lower limb arterial embolism after TKA is extremely rare. A literature review involving 9022 patients who underwent TKAs at the Mayo Clinic and reported that only 3 patients were diagnosed with arterial complications post-operatively ${ }^{[1]}$. In another study involving 4097 patients who underwent TKAs in a Pennsylvania hospital, there were only 7 patients who developed acute arterial ischemia of the lower extremity, with an incidence of only $0.03 \%-0.17 \%{ }^{[2]}$. The primary symptoms include artery thrombosis, arteriovenous fistula, aneurysm formation, and arterial rupture ${ }^{[3]}$. Among them, popliteal artery thrombogenesis is the most common and accounts for $65.9 \%$ of the post-operative complications ${ }^{[4]}$. Orthopedists should be vigilant for acute popliteal artery thrombosis, despite the low incidence because it may cause severe outcomes, such as the need for an amputation. We report a case of acute popliteal artery thrombogenesis after a TKA. Even though the orthopedists and vascular surgeons took active measures in a timely fashion, the patient still had lower limb dysfunction.

\section{Case Report}

The patient, a 64-year-old male, had 38 years of post-traumatic pain in the left knee joint, which had worsened over the past 3 years, thus limiting mobility. The results of X-ray while weight-bearing showed severe osteoarthritis of the left knee with bilateral joint space stenosis (Fig. 1). He underwent a subtotal gastrectomy for gastric perforation 30 years ago. He had no history of hypertension, diabetes, coronary heart disease, cigarette smoking, or alcohol consumption. The physical examination showed that the knee joint was slightly deformed, the range of joint activity was $0^{\circ}-120^{\circ}$, the dorsal foot and posterior tibial artery pulses were good, and the skin temperature was normal. There were no abnormal changes on 
routine blood testing pre-operatively. No abnormalities were noted o color Doppler ultrasound of the lower extremity, electrocardiogram (ECG), and chest X-ray pre-operatively.

The patient underwent a left TKA under general anesthesia. After anesthesia was established, the patient was placed in the supine position, and the surgical field of the left knee joint was sterilized with iodine and alcohol, then a sterile drape was applied. The TKA was initiated with a tourniquet that was inflated to a pressure of $280 \mathrm{mmHg}$. The incision of the left knee was approximately $16 \mathrm{~cm}$ in length, starting from the junction of the tendon and abdomen above the patella and ending $1 \mathrm{~cm}$ inside the tibial tubercle. The skin and subcutaneous tissue were incised from the junction of the rectus femoris and medial femoris, the tendon, joint capsule, and medial patellar retinaculum along the medial patella. There was no effusion in the joint cavity, minimal synovial hyperplasia, and third-degree deterioration of the patellofemoral joint. The medial compartment and meniscus the of knee joint were severely atrophic and some portions of the meniscus were absent. The subchondral bone of the medial condyle of the femur and tibial plateau were exposed and the deterioration of the lateral compartment of the knee joint was slightly less than the medial compartment. The synovium and part of the fat pad under the patella were removed. The periosteum was incised $1 \mathrm{~cm}$ from the medial tibial tubercle and the medial articular capsule was initially released. The osteophytes of femur and tibia, hyperplastic synovium, meniscus, and anterior and posterior cruciate ligaments were removed. The tibial plateau was lifted with a Homann retractor, and the residual synovium, meniscus, and osteophytes were cleared. The posterior internal side of the joint capsule and semimembranosus tendon were loosened. The internal aspect of the accessory ligament was protected. A tibial extramedullary locator and an osteotomy device were mounted. The tibial osteotomy device was mounted at the proximal tibia and located along the internal aspect of the plateau vertical to the tibial force line. The tibial plateau osteotomy plate was located at $0^{\circ}$ and the osteotomy was performed with a $3^{\circ}$ back inclination. The articular surface of the tibial plateau was removed with a swing saw and the edge of the plateau was trimmed. The size of the tibial plateau prosthesis was measured as a No. 3. A distal femur osteotomy was performed with a localizer. The size of the distal femoral prosthesis was measured as a No. 2.5. The anterior and posterior condyles, and two oblique osteotomies were performed; the distal osteotomy was $9 \mathrm{~mm}$. Then, a slightly lateral intercondylar osteotomy was performed. The No. 3 prosthesis model and a 10-mm pad were placed on the femoral and tibial sides. After reduction, the force line was noted to be good, the joint tightness was appropriate, the knee joint was flexed and extended, and the space between flexion and extension was appropriate. The test mold was removed and the incision was irrigated, the bone cement was adjusted, and recording of the time was started. Tibia and femur prostheses were placed. A molecular polyethylene meniscus liner, $10 \mathrm{~mm}$ in height, was placed and held in a straight position for $15 \mathrm{~min}$. At the same time, the patella was denervated, the marginal osteophytes were removed, and the joint surface was repaired. The patella was tested to be satisfactory after bone cement fixation. The wound was irrigated with a large amount of normal saline and the knee joint was restored. The force line was tested to be at the median of the patella and the bending angle of the joint was $130^{\circ}$ and the straightening angle was $0^{\circ}$. The instruments and surgical dressing counts were correct and the joint capsule of the internal side of the patella, aponeurosis, subcutaneous tissues, and skin were gradually sutured along with loosening of the 
tourniquet and hemostasis by compression for $10 \mathrm{~min}$. The wound was bent up. The left lower limb was bent up with an elastic bandage. The operation was successful with approximately $300 \mathrm{ml}$ of hemorrhage intra-operatively. After palpation, pulsation of dorsalis pedis artery was normal and the blood supply of the toe tip was good.

Six hours after surgery the patient complained of slight swelling accompanied by mild pain in the left lower limb. The physical examination revealed that the skin temperature of the left lower extremity was decreased and the tingling sensation of the lateral leg and dorsal foot were slightly decreased. The pulse of the dorsalis pedis artery was audible by Doppler, but the pulsation was weak and movements of the toe and ankle joint were good. Hence, the wound elastic bandage was loosened and the patient felt better. Approximately $16.5 \mathrm{~h}$ after surgery, the patient complained of increased swelling in the left lower limb. The skin temperature was decreased, the dorsalis pedis artery pulse was absent, and the toe dorsal stretch force was weak. Ultrasonography and angiography of the left lower extremity arteriovenous were performed. The results showed that the blood flow in the lower segment of the left popliteal artery was interrupted and a thrombus has formed (Fig. 2).

The patient was returned to the operating room for a popliteal artery exploration and the popliteal artery was stiff with negative elasticity. The vascular adventitia was complete and a thrombus was considered. The popliteal arterial thrombus was clamped using a vascular clamp and a 1-cm wound was incised longitudinally at the proximal and distal arteries. Segments of the thrombus, 2.5 and $3 \mathrm{~cm}$ in length, were removed from the incision (Figs. 3 and 4). The vessels were washed with heparin saline and no residual thrombus remained. The arterial incision was sutured using $8-0$ prolene and the vascular clamp was loosened. The artery filled and pulsed, and the dorsalis pedis and posterior tibial arteries were palpable. The skin temperature of the left lower limb rebounded. A fasciotomy and relaxation were performed to prevent compartment syndrome. A post-operative vascular color ultrasound of the lower limb showed favorable blood flow. Considering the patient's mood and economic factors, angiography of the lower limb was not performed. Seven months after the operation the motion of the patient's left knee joint was $0^{\circ}-110^{\circ}$. In addition, the patient had anterolateral leg numbness and foot drop.

\section{Discussion}

There are few reports on artery injuries after TKAs. The four primary causes are as follows. (1) The patient has basic vascular diseases concurrently, such as atherosclerosis, diabetes, and lower arterial occlusion, which lead to vascular complications. (2) A tourniquet is used during the TKA, which causes a vascular embolism and insufficient blood supply; however, it mainly develops at the superficial femoral artery. Loosening the tourniquet will cause stripping of the endarterium and slow the blood flow, thus leading to secondary onset of embolism and worsened blood supply insufficiency ${ }^{[7]}$. (3) For patients with severe flexion deformity due to the contracture of posterior joint capsule and popliteal vessel, the popliteal artery will be extracted after deformity correction, which may also cause injury to the endarterium ${ }^{[8]}$. In addition, arterial extraction, corrosion of vessels, bones, and tendons may also lead to 
injury. (4) The vessels are directly punctured or cut off by the medical saw, scalpel, drag hook, or concrete block during the operation.

Rui He et al. reviewed 41 reports and summarized the characteristics and treatment methods of artery embolism. The incidence of popliteal artery involvement was the highest, accounting for $80 \%$ of all cases [9]. The patients will encounter a devastating outcome (knee amputation) if the arterial blood flow fails to be restored. In our case, the embolism nearly occupied the entire popliteal artery. The exploration showed continuous existence and no contrast agent exostoses were seen. Therefore, a transection injury by the saw was excluded. A possible reason for the popliteal artery thrombus might be a Homann hook injury on the posterior tibial plateau when inserting the hook or application of the tourniquet. Ninomiya ${ }^{[13]}$ has performed arteriography on deceased human bodies and found that the risk of a popliteal artery injury increased if the hook was inserted too deep into the posterior knee joint or inserted over $1 \mathrm{~cm}$ into the soft tissue. Another possibility that may cause thrombosis is that the atrial plaque at the lower limb is broken and bleeding, which may lead to inner wall swelling in the vascular cavity and slow blood flow. In addition, the blood will be in a hypercoagulable state by infusing red blood cells during the operation. As a result, thrombogenesis is formed; however, it was unfortunate that we did not perform arterial ultrasonic Doppler pre-operatively, which suggests that we had more to do with respect to peri-operative management.

There are several treatment methods for artery embolism, including interventional thrombolytic therapy, transvenous catheter embolectomy, artery bypass reconstruction, and vascular implantation and

anastomosis $[2,4,5,6,10,11]$. Catheter embolectomy was the most extensively used. Kobayashi et al. [6] reported one case of applying urokinase + prostaglandin to achieve embolism dissolution and revascularization after TKA, which resulted in a good treatment effect. Early diagnosis and treatment are the key for restoration of blood supply. In our case we used prolinase to dissolve the embolus when we determined the symptoms of popliteal artery thrombosis and lower limb ischemia, and we immediately consulted micro-surgeons to perform popliteal artery exploration after the embolectomy failed. As a result, although the blood circulation was recovered, the patient developed complications, including stiffness of the affected limb and foot drop due to long-term ischemia.

\section{Conclusion}

There are very few reports on artery embolism after TKA. Although the incidence is low, it will increase with the aging trend and the increase in number of TKAs. Our experience was as follows. For elderly patients, especially those who have peripheral vascular disease, pre-operative examinations must be complete and the operative risks should be evaluated. During the operation, the application of tourniquets must be applied with care. The operation should be gentle to avoid iatrogenic injury. When a vascular injury is suspected, lower vein angiography or color ultrasound B should be performed in a timely fashion to clarify the diagnosis. If a thrombus is confirmed, appropriate treatment should be undertaken. Although popliteal artery exploration is intuitive, it may cause adhesions and infections of the posterior 
knee joint. Patients should be guided to engage in active rehabilitative exercises after a TKA to prevent thromobogenesis.

\section{Abbreviations}

TKA

Total knee arthroplasty

\section{Declarations}

\section{Acknowledgements}

None.

\section{Authors' contributions}

The authors are grateful for the help from Mr Wei for the linguistic support in producing this manuscript,.The authors were wholly responsible for all measurements, scientific and clinical evaluations, and the content of this manuscript.Ri Liu collated the information on our patients from the case notes, structured the manuscript,reviewed the literature and defined the content of the discussion. Lishu Gao and Kunnan Xie consulted with our patients, and input into the discussion. All authors have read and approved the final manuscript.

\section{Funding}

None.

\section{Availability of data and materials}

The datasets used and/or analyzed during the current study are available from the corresponding author on reasonable request.

\section{Ethics approval and consent to participate}

This study was approved by the ethics committee of The second hospital of TangShan. Written informed consent was obtained from the patient.

\section{Consent for publication}

Not applicable.

\section{Competing interests}

The authors declare that they have no competing interests. 


\section{Author details}

1 The second hospital of Tangshan, 21 North Jianshe road, Tangshan, China 063000.

2 The people's hospital of Tangshan, 65 Shengli road, Tangshan, China 063000.

\section{References}

[1] Rand JA. Vascular complications of total knee arthroplasty. Report of three cases. J Arthroplasty 1987; 2: 89.

[2] Calligaro KD, deLaurentis DA, Booth RE, et al. Acute arterial thrombosis associated with total knee arthroplasty. J Vasc Surg 1994; 20: 927.

[3] Langkamer VG. Local vascular complications after knee replacement: a review with illustrative case reports. Knee 2001; 8: 259.

[4] Holmberg A, Milbrink J, Bergqvist D. Arterial complications after knee arthroplasty: 4 cases and a review of the literature. Acta Orthop Scand 1996; 67: 75.

[5] Ohira T, Fujimoto T, Taniwaki K. Acute popliteal artery occlusion after total knee arthroplasty. Arch Orthop Trauma Surg, 1997; 116(6-7): 429-430.

[6] Kobayashi S, Isobe K, Koike T, et al. Acute arterial occlusion associated with total knee arthroplasty. Arch Orthop Trauma Surg, 1999; 119(3-4): 223-224.

[7] Kumar SN囚Chapman JA囚Rawlins I.Vascular injuries in total knee arthroplasty. A review of the problem with special reference to the possible effects of the tourniquet. J Arthroplasty, 1998; 13(2): 211-216.

[8] Dawson AG, Bachoo P, Sutherland AG. Does knee replacement surgery lead to chronic limb ischemia? J Knee Surg, 2010; 23(4): 223-228.

[9] Rui He, Liu Yang Acute arterial occlusion in the midpiece of femoral artery following total knee arthroplasty: Report of one case. Chinese Journal of Traumatology, 2016; 19: 116-118.

[10] Berger C, Anzbock W, Lange A, et al. Arterial occlusion after total knee arthroplasty. J Arthroplasty, 2002; 17(2): 227-229.

[11] Gregory PC, Rogic R, Eddington C. Acute arterial occlusion after total knee arthroplasty. Am J Phys Med Rehabil, 2006; 85(11): 924-926.

[12] Kehagias, E., loannou, C. V., et al. Intraoperative Endovascular Stent-graft Repair of a Popliteal Artery Laceration and Occlusion during Total Knee Arthroplasty. Annals of Vascular Surgery, 2015; 29(7): 1453.9-1453.14. 
[13] Ninomiya JT, Dean JC, Goldberg VM. Injury to the popliteal artery and its anatomic location in total knee arthroplasty. J Arthroplasty, 1999; 14(7): 803-809.

\section{Figures}
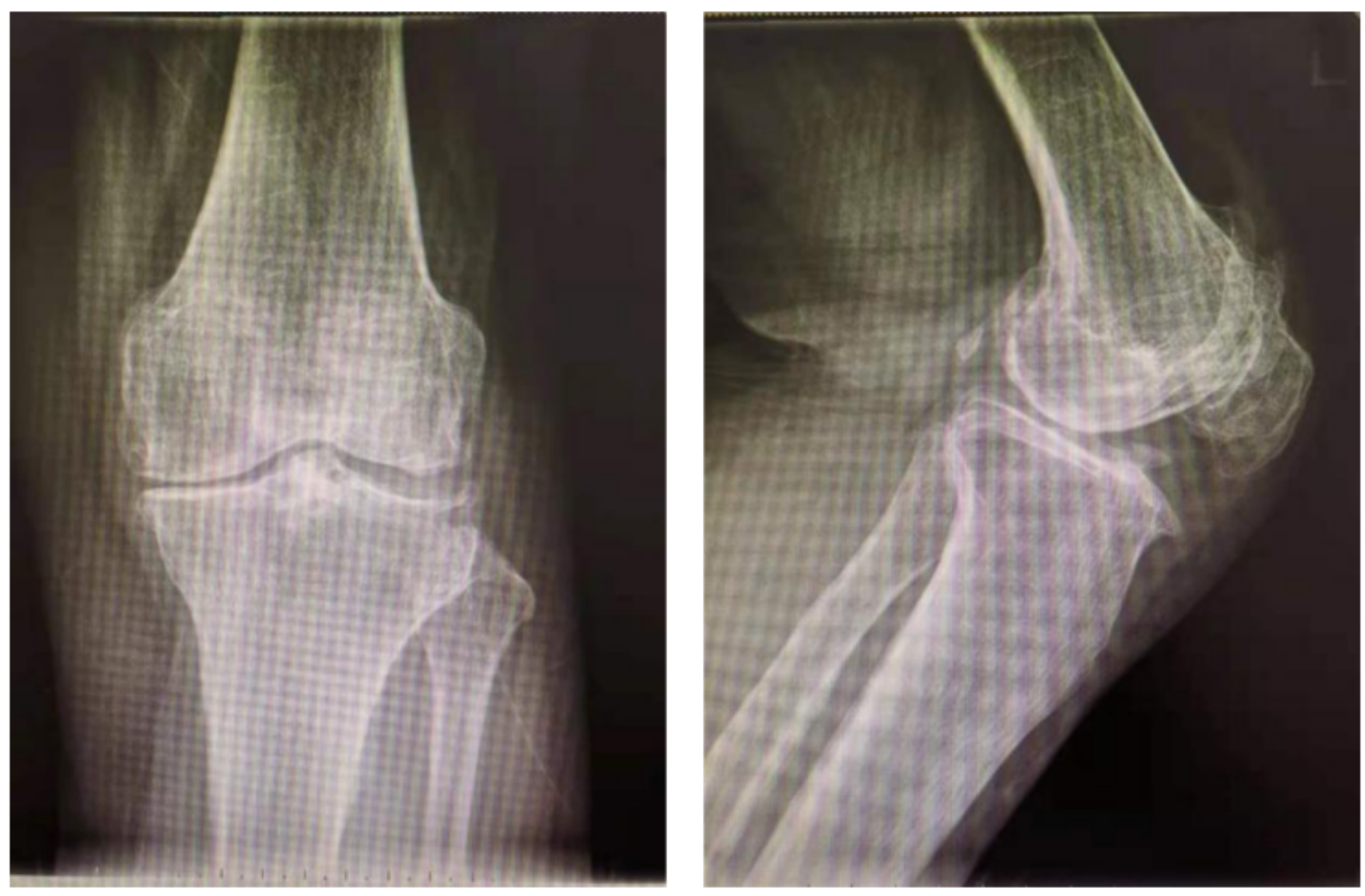

\section{Figure 1}

The results of X-ray while weight-bearing before surgery. 


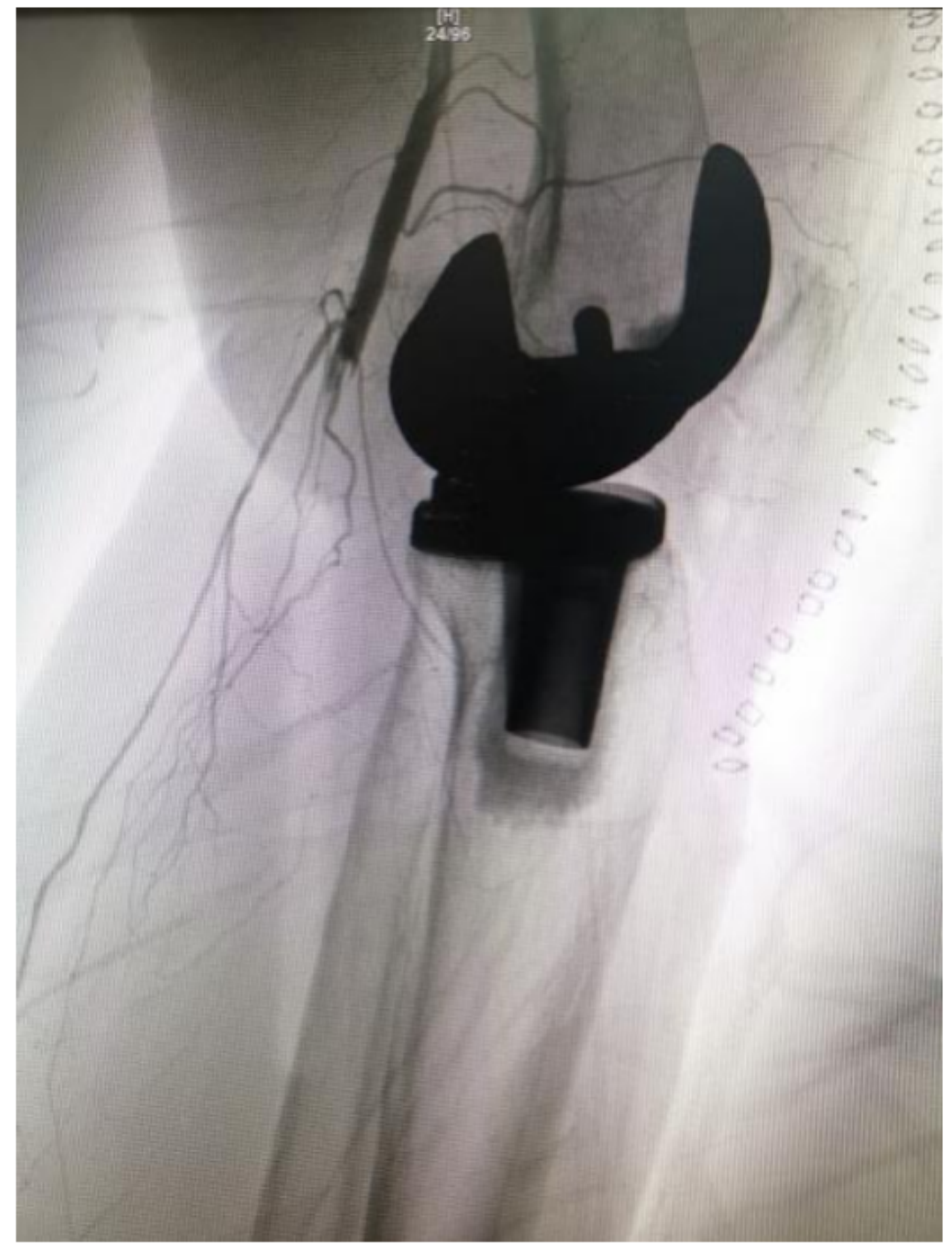

\section{Figure 2}

Arteriovenous angiography of the left lower limb after surgery. 


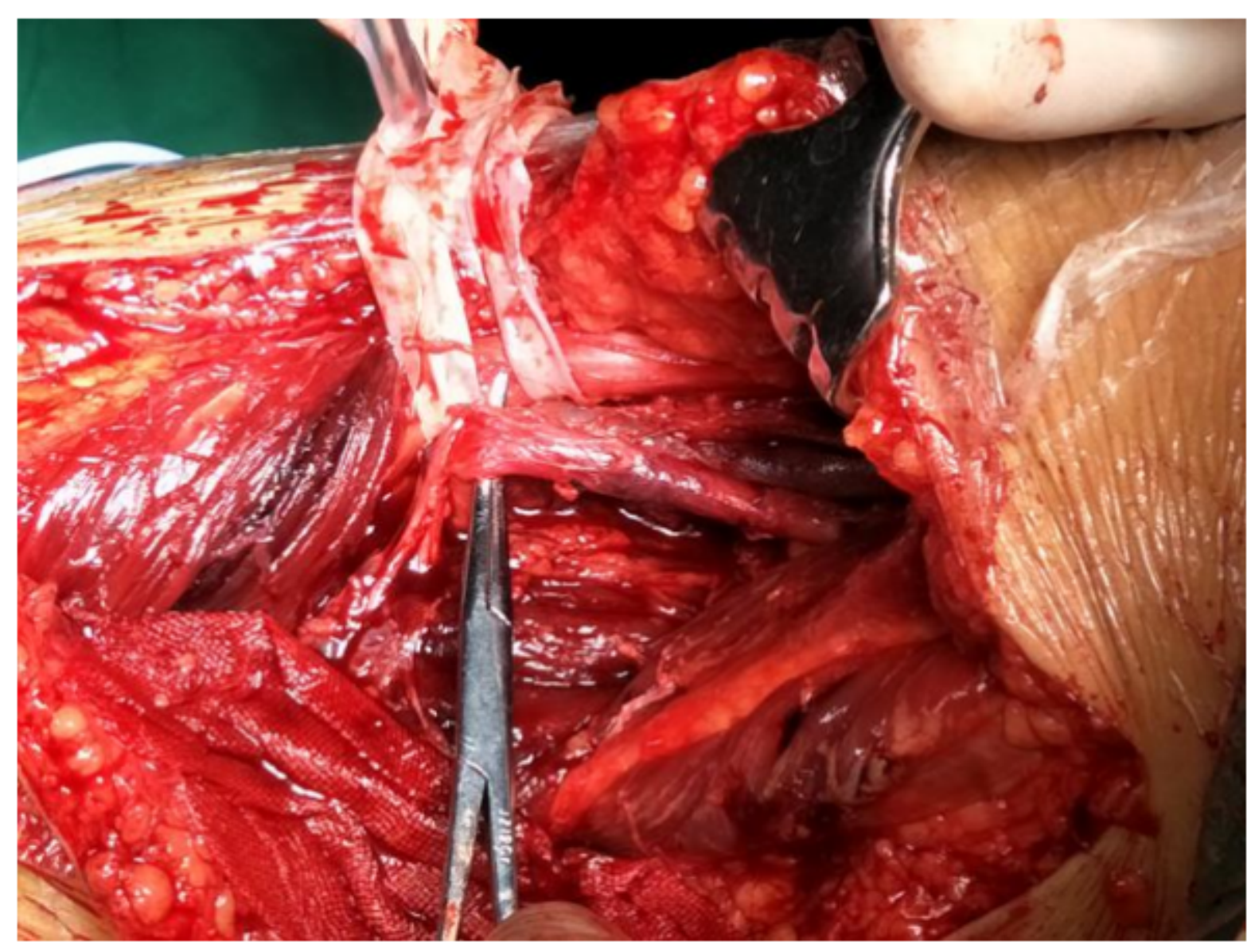

Figure 3

The second surgical procedure. 


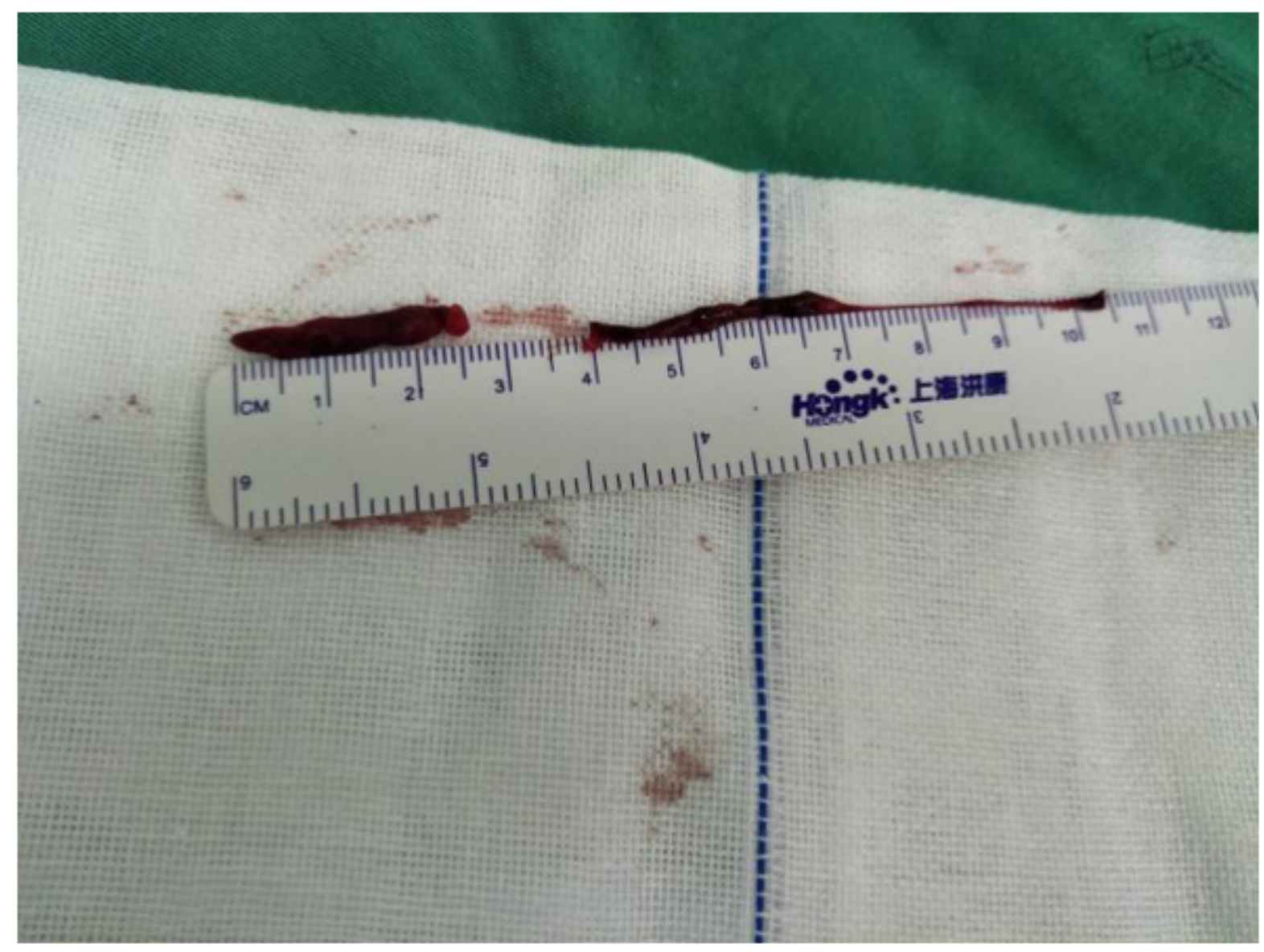

Figure 4

Two thrombi removed after the second operation. 